\title{
Hippocampal Atrophy in Major Depression: a Function of Childhood Maltreatment Rather than Diagnosis?
}

\author{
Nils Opel', Ronny Redlich', Peter Zwanzger', Dominik Grotegerd', Volker Arolt', Walter Heindel', \\ Carsten Konrad ${ }^{3}$, Harald Kugel ${ }^{2,4}$ and Udo Dannlowski, ${ }^{*, 1,3,4}$ \\ 'Department of Psychiatry, University of Münster, Münster, Germany; ${ }^{2}$ Department of Clinical Radiology, University of Münster, \\ Münster, Germany; ${ }^{3}$ Department of Psychiatry, University of Marburg, Münster, Germany
}

\begin{abstract}
Reduced hippocampal volumes are probably the most frequently reported structural neuroimaging finding associated with major depressive disorder (MDD). However, it remains unclear whether altered hippocampal structure represents a risk factor for or a consequence of MDD. Reduced hippocampal volumes were consistently reported in subjects affected by childhood maltreatment. As the prevalence of childhood maltreatment is highly elevated in MDD populations, previous morphometric findings regarding hippocampal atrophy in MDD therefore might have been confounded by maltreatment experiences. The aim of this study was to differentiate the impact of childhood maltreatment from the influence of MDD diagnosis on hippocampal morphometry. Depressed patients (85) as well as 85 age- and sex-matched healthy controls underwent structural MRI. The Childhood Trauma Questionnaire was administered to estimate experiences of childhood maltreatment. Hippocampal volume and surface structure was examined by the use of two independent methods, automated segmentation (FSL-FIRST) and voxel-based morphometry (VBM8). In line with existing studies, MDD patients showed reduced hippocampal volumes, and childhood maltreatment was consistently associated with hippocampal volume loss in both, patients and healthy controls. However, no analysis revealed significant morphological differences between patients and controls if maltreatment experience was regressed out. Our results suggest that hippocampal alterations in MDD patients may at least partly be traced back to higher occurrence of early-life adverse experiences. Regarding the strong morphometric impact of childhood maltreatment and its distinctly elevated prevalence in MDD populations, this study provides an alternative explanation for frequently observed limbic structural abnormalities in depressed patients.
\end{abstract}

Neuropsychopharmacology (20I4) 39, 2723-273I; doi:I0.I038/npp.20I4.I45; published online 9 July 2014

\section{INTRODUCTION}

Major depressive disorder (MDD) is one of the most debilitating diseases worldwide, and the social and economic burden is a major challenge in public health (World Health Organization, 2001). As effective treatment and prevention requires knowledge of risk factors and their neurobiological mechanisms, there is a need for a more detailed understanding of the etiology and the biological implications of this disease.

MDD and other affective disorders were consistently shown to be accompanied by functional and structural aberrations in cerebral areas involved in emotion processing (Cusi et al, 2012; Stuhrmann et al, 2011). Neuroimaging studies on MDD showed various alterations in limbic structures, including the hippocampus, the striatum, and

\footnotetext{
*Correspondence: Professor U Dannlowski, Department of Psychiatry, University of Münster, Albert-Schweitzer-Strasse II, Münster 48I49, Germany, Tel: +49 25। 835660I, Fax: +49 25I 83566।2,

E-mail: dannlow@uni-muenster.de

${ }^{4}$ These authors contributed equally to this work.

Received 6 December 2013; revised 8 February 2014; accepted 15 April 2014; accepted article preview online 13 June 2014
}

the cingulate cortex (Arnone et al, 2012a). In fact, reduced hippocampal volume is probably the most frequently reported finding in neuroimaging studies comparing MDD patients with healthy controls (Cole et al, 2011). Recent meta-analyses underlined this notion, but the debate on its underpinnings prevails (MacQueen and Frodl, 2011). Alterations in this structure have been found to be associated with various clinical and demographic characteristics in patient samples (McKinnon et al, 2009). However, some studies indicate that reduced hippocampal volumes might be apparent before onset of disease in subjects at risk for depression (Amico et al, 2011). Hence, hippocampal alterations have been suspected to mediate the effect of longterm stressful life events on depression risk rather than constituting a disease marker (Frodl et al, 2010b).

The hippocampus is one of the most plastic structures of the human brain, and genesis of hippocampal neurons has been shown to occur life-long and to be actuated by environmental as well as by genetic influence (Covic et al, 2010; Eriksson et al, 1998). Thus, impaired hippocampal neurogenesis and synaptic dysfunction are susceptible to have a key role in the pathophysiology of depressive disorder (Duman and Aghajanian, 2012). As its pronounced neuroplastic capacity is influenced by elevated levels of 
glucocorticoids typically involved in stress regulation, gray matter loss may be induced by adverse experiences (Wang et al, 2010). The detrimental impact of stressful life events seems to be markedly increased in decisive periods of brain development during childhood (Heim et al, 2008).

Consequently, traumatic early-life experiences have been shown to exacerbate transformation processes of cerebral structures linked to emotional regulation (van Harmelen et al, 2010; Teicher et al, 2004). Small hippocampal volumes have been repeatedly detected in subjects affected by childhood maltreatment (Dannlowski et al, 2012; Teicher and Samson, 2013). There has been growing evidence regarding the genesis of these trauma-related changes in limbic structures to be independent from disease status, as they could be found in both healthy subjects and currently depressed patients suffering from MDD (Chaney et al, 2014; Edmiston et al, 2011; Frodl et al, 2010b; Vythilingam et al, 2002). Teicher et al (2012) showed that hippocampal shape alterations in subjects having experienced childhood maltreatment is not mediated by the presence or absence of life-time affective disorders.

Childhood maltreatment is a major risk factor for the development of affective disorders (Gilbert et al, 2009) and unsurprisingly, its prevalence is highly elevated in MDD populations (Scott et al, 2012). Furthermore, adverse early-live experiences have been shown to aggravate course and outcome of MDD (Nanni et al, 2012). As childhood maltreatment appears to show a massive impact on hippocampal volume and structure in patients and controls alike, it could represent a powerful confounder for a direct comparison of these groups regarding hippocampal size and structure. Unfortunately, to the best of our knowledge, there is no study available demonstrating hippocampal atrophy in depressed patients that systematically controlled for these detrimental effects of childhood maltreatment.

Regarding the dwelling dispute on either the state (disease marker) or trait (risk factor) characteristic of hippocampal structure, it appears exigent to investigate the structural impact of both early-life adversities and current disease status. Therefore, in our study, we sought to clarify to which level hippocampal changes represent effects of current disease or long-term consequences of adverse experiences in earlier life. The impact of childhood maltreatment on hippocampal alterations was investigated in a large sample of 85 currently depressed patients and 85 healthy controls. We expected hippocampal volumes in both groups to be influenced by childhood maltreatment experiences. We further hypothesized that differences in hippocampal morphometry between patients and healthy controls would be diminished or even not be traceable when accounting for childhood maltreatment.

\section{MATERIALS AND METHODS}

\section{Subjects}

Currently depressed patients (85) as well as 85 age- and gender-matched healthy controls participated in our study. All patients were under inpatient treatment at the University Hospital of Münster. Healthy subjects were recruited via newspaper advertisement, with no apparent
Table I Sociodemographic and Clinical Characteristics of our Study Sample Consisting of 85 Currently Depressed Patients and 85 Healthy Controls

\begin{tabular}{|c|c|c|c|}
\hline & $\begin{array}{l}\text { Healthy controls } \\
\quad(N=85)\end{array}$ & $\begin{array}{l}\text { MDD patients } \\
\quad(N=85)\end{array}$ & $P$-value \\
\hline & Mean \pm SD & Mean \pm SD & \\
\hline Age & $37.2 \pm 11.8$ & $37.6 \pm 12.0$ & 0.847 \\
\hline $\operatorname{Sex}(m / f)$ & $34 / 51$ & $31 / 54$ & 0.752 \\
\hline $\begin{array}{l}\text { Occupational education } \\
\text { (years) }\end{array}$ & $3.1 \pm 1.7$ & $3.1 \pm 1.3$ & 0.816 \\
\hline CTQ total & $34.0 \pm 7.4$ & $44.8 \pm 15.9$ & $<0.001$ \\
\hline Emotional neglect & $9.2 \pm 3.3$ & $13.0 \pm 5.3$ & $<0.001$ \\
\hline Physical neglect & $6.7 \pm 2.1$ & $7.5 \pm 2.6$ & 0.024 \\
\hline Sexual abuse & $5.0 \pm 0.2$ & $6.4 \pm 3.9$ & 0.001 \\
\hline Physical abuse & $5.8 \pm 2.1$ & $7.0 \pm 3.3$ & 0.005 \\
\hline Emotional abuse & $7.3 \pm 2.8$ & $10.8 \pm 5.3$ & $<0.001$ \\
\hline HAM-D & $1.2 \pm 1.6$ & $23.4 \pm 5.4$ & $<0.00$ I \\
\hline BDI & $2.2 \pm 3.0$ & $29.2 \pm 9.4$ & $<0.001$ \\
\hline $\begin{array}{l}\text { Duration of illness } \\
\text { (months) }\end{array}$ & NA & $94.3 \pm 100.6$ & NA \\
\hline Number of episodes & NA & $3.6 \pm 3.5$ & NA \\
\hline
\end{tabular}

Means, $\pm \mathrm{SD}$, and group differences (as measured with $t$-tests or $\chi^{2}$-test).

Table 2 Medication of Patients Under Antidepressant Drug Therapy $(n=8 \mathrm{l})$

\begin{tabular}{lc}
\hline Medication type & $\begin{array}{c}\text { Number of } \\
\text { patients }\end{array}$ \\
\hline SSNRI & 66 \\
SSRI & 19 \\
SNDRI & 3 \\
SNRI & 2 \\
Tricyclic antidepressants & 5 \\
MAO-inhibitor & 10 \\
Mood-stabilizer & 8 \\
Antipsychotics & 36 \\
\hline
\end{tabular}

link to childhood maltreatment indicated. Clinical diagnosis were obtained using the DSM-IV Structured Clinical Interview (SCID-I) in patients and controls (Wittchen et al, 1997). The Hamilton Rating Scale for Depression (HAM-D) (Hamilton, 1960), and the Beck Depression Inventory (BDI) (Beck and Steer, 1987) were administered to assess the current level of depression. Only acutely depressed patients showing scores of $>18$ and healthy controls showing scores of $<10$ in the BDI were included in our study (see Table 1 for details). Most depressed subjects were medicated (see Table 2 for details). In order to evaluate the potential impact of antidepressant treatment, medications were coded in terms of dose and treatment durations into levels from 1 to 4 according to the suggestions of Sackeim (2001) as we and others have conducted previously (Dannlowski et al, 2009; Surguladze et al, 2005; Suslow et al, 2010). Exclusion criteria 
were any history of severe neurological (eg, concussion, stroke, tumor, neuro-inflammatory diseases) and medical (eg, cancer, chronic inflammatory or autoimmune diseases, infections) conditions. Controls were ensured to be free from any history of psychiatric disorders or any psychotropic medication. Occurrence of childhood maltreatment was evaluated with the Childhood Trauma Questionnaire (CTQ) assessing five types of adverse early-life experiences by means of a 25 -item retrospective self-report questionnaire (Bernstein et al, 1994).

The Ethics Committee at the University of Münster approved the study, and written informed consent was obtained from all participants.

\section{Image Acquisition}

T1-weighted high-resolution anatomical images of the head were acquired using a three-dimensional (3D) fast gradient echo sequence (Turbo field echo), with a repetition time of $7.4 \mathrm{~ms}$, echo time $=3.4 \mathrm{~ms}$, flip angle $=9^{\circ}$, two signal averages, inversion prepulse every $814.5 \mathrm{~ms}$, acquired over a field of view of 256 (feet-head) $\times 204$ (anteriorposterior) $\times 160$ (right-left) $\mathrm{mm}$, phase encoding in AP and RL direction, reconstructed to voxels of $0.5 \mathrm{~mm} \times$ $0.5 \mathrm{~mm} \times 0.5 \mathrm{~mm}$. These high-resolution images were processed with two independent morphometric methods-an automated segmentation procedure and a voxel-based morphometric approach. Both methods can be regarded complementing each other. Although automated segmentation uses pre-defined anatomical landmarks to delineate the hippocampus boundaries in order to obtain volume and shape information, voxel-based morphometry focusses on gray matter structure after segmentation of gray matter across the entire brain and normalization to a template, allowing voxel-wise statistics on the determined gray matter values. Both procedures are highly reliable and sensitive methods for hippocampus morphometry in neuropsychiatric disorders, including MDD (Bergouignan et al, 2009; Lai and $\mathrm{Wu}, 2011)$.

\section{Automated Hippocampus Segmentation with FSL-FIRST}

Segmentations of the hippocampus were generated using the FIRST algorithms. FIRST is a fully automated segmentation software included in the FMRIB Software Library (FSL, version 5.0.0) providing analyzing tools not only for overall volume information of the segmented structures but also for localized differences in shape. The segmentation is based on shape and appearance models constructed from 336 manually segmented images using Gaussian assumptions combined with a Bayesian probabilistic approach (http://fsl. fmrib.ox.ac.uk/fsl/fsl-4.1.9/first/index.html). Surface meshes representing the volumetric information of each structure are formed using parameterized deformable surfaces (Nugent et al, 2012). FIRST then ascertains the most probable shape by means of linear combinations of shape variations employing the learned models. Moreover, the segmentation process includes boundary correction and registration to a MNI152 template using 12 degrees of freedom as well as a subcortical mask to detect and eliminate voxels outside the subcortical structure. Quality of segmentation and registration were manually checked for each subject. Volumes of the bilateral hippocampi were computed and further analyzed using the SPSS software package in three steps:

(1) To investigate differences in hippocampal volumes between patients and controls, an ANCOVA was performed with group (patients $v s$ controls) as the between-subjects factor, laterality (left $v s$ right) as the within-subjects factor, and intracranial volume (ICV) as a nuisance covariate. We hypothesized that in accordance with numerous previous neuroimaging studies, MDD patients would show reduced hippocampal volumes compared with healthy controls.

(2) To investigate the impact of maltreatment on hippocampal morphometry, partial correlations of hippocampal volumes with CTQ-scores were performed, controlling for ICV. This was done for the entire sample $(N=170)$, as well as within each group separately. We hypothesized that we could replicate previous findings that hippocampal volumes are negatively correlated with childhood maltreatment severity in both groups separately (and also in the entire combined sample).

(3) In order to explore competing effects of group and childhood maltreatment, a second ANCOVA was performed identical to step 1, but now additionally controlling for CTQ-scores. We hypothesized that group differences between MDD and healthy controls are strongly diminished or even disappear if childhood maltreatment is regressed out. In addition, hierarchical regression analyses were performed including age, gender, ICV, and group membership in step 1, adding CTQ-scores in step 2 and vice versa, including age, gender, ICV, and CTQ-scores in step 1, adding group membership in step 2.

Finally, for visualization purposes, the localization of shape differences were computed using surface-based vertex analysis, which is based on multivariate statistics yielding a 3D mesh of the structure of interest. Thus, group differences and correlation of CTQ with alterations in hippocampal shape were visualized.

\section{VBM}

The VBM8-toolbox (http://dbm.neuro.uni-jena.de/vbm) was used for analyzing gray matter information of the hippocampus, as described in our previous work (Dannlowski et al, 2012) (see Supplementary Materials and Methods for details).

\section{RESULTS}

\section{FSL-FIRST}

(1) Consistent with our hypothesis, a main effect of the group emerged, $F(1,167)=5.03, P=0.027, \eta_{\mathrm{p}}^{2}=0.029$, indicating smaller hippocampal volumes in MDD patients compared with healthy controls. No main effect or interaction of laterality occurred (all $P>0.3$ ).

(2) Correlation analyses in the entire sample revealed significant negative correlations of bilateral hippocampal 
volumes and CTQ-scores, controlling for ICV (left: $r_{\mathrm{p}}=$ $-0.321, P<0.001$; right: $\left.r_{\mathrm{p}}=-0.272, P<0.001\right)$. All CTQ subscales showed widely similar effects on hippocampal volume (see Supplementary Table S1 for details). We further checked whether these associations were influenced by other relevant variables by adding age, gender, group membership, education, HAM-D, and BDI as control variables. However, still highly significant correlations were detected (left: $r_{\mathrm{p}}=-0.250, P=0.001$; right: $r_{\mathrm{p}}=-0.218$, $P=0.005$ ).

Separate correlation analyses in patients and healthy controls alone revealed widely the same effects. Both groups showed smaller hippocampal volumes with increasing CTQ-scores (MDD patients: left: $r_{\mathrm{p}}=-0.283, P=0.009$; right: $r_{\mathrm{p}}=-0.278, P=0.010$; healthy controls: left: $r_{\mathrm{p}}=$ $-0.245, P=0.025$; right: $\left.r_{\mathrm{p}}=-0.252, P=0.021\right)$.

In the patient sample, there were no significant associations of hippocampal volumes and number of depressed episodes, duration of illness or medication load scores controlling for ICV, nor were there any significant associations between childhood maltreatment and medication load, number of depressed episodes or duration of illness controlling for ICV (all $P>0.05$ ).

(3) When accounting for CTQ-scores as additional covariate in the ANCOVA from step 1, no significant group differences could be observed anymore, $F(1,166)=0.33$, $P=0.565, \eta_{\mathrm{p}}^{2}=0.002$. However, a strong effect of CTQ emerged also in this analysis $F(1,166)=15.86, P<0.001$, $\eta_{\mathrm{p}}^{2}=0.089$, as could be expected from the results above. Adding the interaction term of $\mathrm{CTQ} \times$ Diagnosis to the design did not alter the pattern of results and did not yield a significant interaction, $F(1,163)=0.77, P=0.38$. No other main effect or interaction reached significance (all $P>0.4$ ).

Hierarchical regression analyses revealed a significant increase in explained variance when additionally accounting for CTQ-scores (left: $R^{2}$-change $=0.054, F$-change $(1,162)=12.825, \quad P<0.001 ; \quad$ right: $\quad R^{2}$-change $=0.057$, $F$-change $(1,162)=11.696, P=0.001)$, vice versa, no significant increase in explained variance could be detected when MDD diagnosis was additionally included in the model (left: $R^{2}$-change $=0.007, F$-change $(1,162)=1.560$, $P=0.213$; right: $R^{2}$-change $<0.001, F$-change $(1,162)=$ $0.014, P=0.907)$.

Finally, FIRST surface-based vertex-wise shape analyses visualize volume reductions in MDD compared with controls (Figure 1a) and the decline of this effect after adding CTQ-scores as covariate (Figure 1b). The vertex analysis further revealed fronto-temporal, occipito-medial, and occipito-temporal areas of the left hippocampus to be significantly negatively correlated with childhood maltreatment in whole-sample analysis (FDR-corrected, $P<0.05$; see Figure 2b). In depressed patients, fronto-temporal and occipito-temporal areas were significantly negatively correlated with childhood maltreatment (FDR-corrected, $P<0.05$; see Figure 2a).

\section{VBM}

Similar to the volumetric finding (and to our previous report on childhood maltreatment using the same method), a main effect of CTQ-scores on hippocampal gray matter volume emerged in the bilateral hippocampus $(x=-14$, $y=-10, z=-24, \mathrm{Z}=3.43, r=-0.26, P=0.0003, k=675$; right: $x=22, \quad y=-27, \quad z=-6, \quad Z=3.29, \quad r=-0.25$, $P=0.0005, k=641$, see Figure 3 ).

The two-sample $t$-test confirmed the volumetric findings yielding bilateral clusters with decreased gray matter volumes in depressed patients compared with healthy controls (left: $x=-20, \quad y=-22, \quad z=-12, \quad Z=3.04$, $P=0.0012, k=1048$ voxels; right: $x=22, y=-13$, $z=-12, \mathrm{Z}=2.92, P=0.0018, k=813$ voxels). However, after adding CTQ-scores to the design as a nuisance regressor, these group differences did not reach significance (all cluster sizes $k<81$ ).

\section{DISCUSSION}

At first sight, our data seem to replicate two well-established findings from different fields of neuroimaging research. First, our finding of reduced hippocampal volumes in depressed patients compared with healthy controls is well in line with numerous imaging studies showing similar effects, including meta-analyses (Arnone et al, 2012a; Cole et al, 2011; MacQueen and Frodl, 2011). Second, the results of our study suggest a strong impact of childhood maltreatment on hippocampal morphometry in both, patients and healthy subjects, with comparable effect sizes in both samples (accounting for $\sim 8-9 \%$ variance of hippocampal volume), which is also in accordance with several previous studies reporting similar effects and effect sizes (Dannlowski et al, 2012; Edmiston et al, 2011; Teicher et al, 2012) but contradicts a study by Lenze et al (2008) that found childhood adversity to be associated with earlier onset of MDD but not with reduced hippocampal volume.

Thus, our present data confirm the neuro-structural influence of childhood maltreatment on hippocampal volume in a large sample of MDD patients and healthy controls. All trauma subtypes showed widely similar effects on hippocampal volume. However, these results should be interpreted with caution, as physical and sexual abuse subscales showed much less variance compared with emotional subscales. Presence and level of depressive symptoms did not change the characteristics of this association. Remarkably, this negative correlation remained significant even when accounting for group membership as a control variable in the whole-sample analysis, as it is also found in both groups separately. This is again well in line with previous reports demonstrating strong maltreatment effects on hippocampal volumes even if MDD patient status or life-time presence of affective disorders is accounted for (Chaney et al, 2014; Teicher et al, 2012).

However, no significant disparity in hippocampal volumes between patients and healthy controls could be discerned anymore after inclusion of CTQ-scores as a covariate in the analysis of group differences. The impact of childhood adversity on hippocampal changes in MDD was also underlined by the significant increase in explained variance, when additionally accounting for CTQ-scores in the hierarchical regression analysis as well as by the fact that, vice versa, no significant increase in explained variance could be observed when additionally including MDD diagnosis in the regression model. Hence, our results might indicate that frequently observed hippocampal 


\section{b}

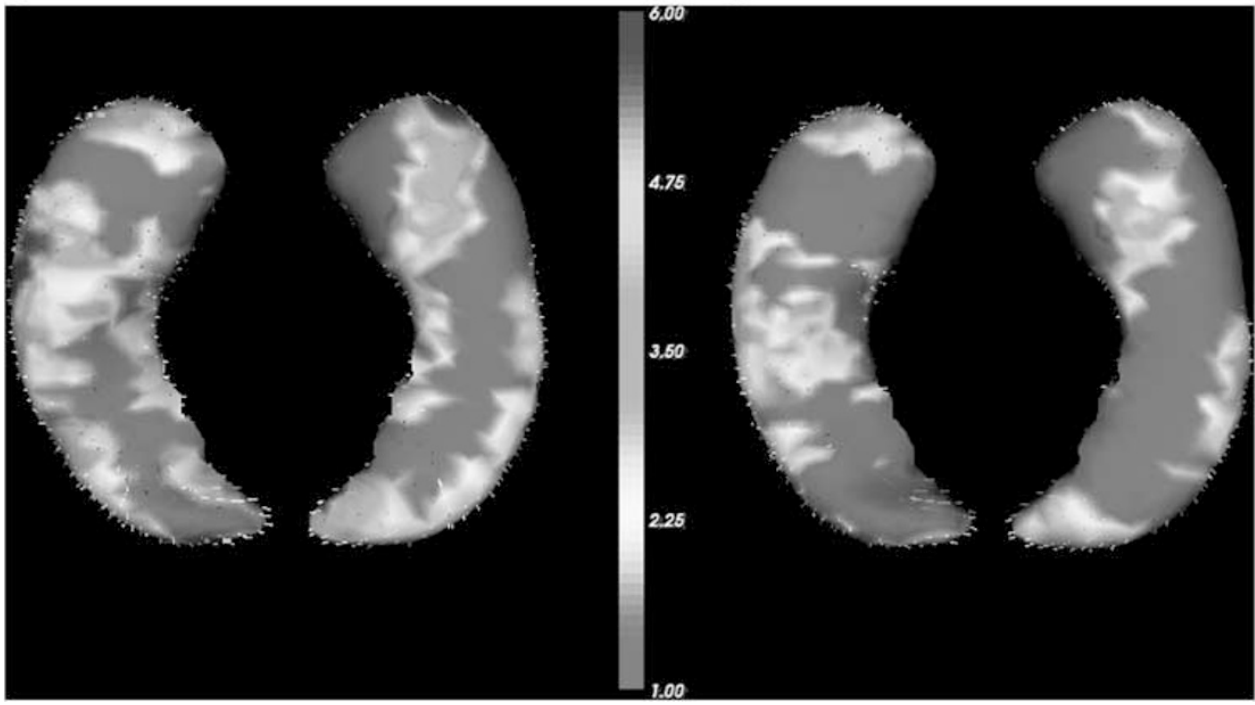

Figure I Results of FSL-FIRST vertex analysis of group differences in left hippocampal shape (dorsal and ventral view). The color bar indicates the values of the F-statistics: an increase from red to blue means transition from lower to higher statistical significance, whereas the length of the vectors displays mean differences between groups. For display reasons, unthresholded values are presented. The vectors indicate the direction of change: vectors pointing inwards represent local atrophy. (a) Major depressive disorder patients show significant local atrophy in fronto-temporal and temporal areas of the left hippocampus compared with healthy controls. (b) Significant differences in hippocampal shape between patients and healthy controls (blue areas) vanish if Childhood Trauma Questionnaire score is included as a covariate.

a

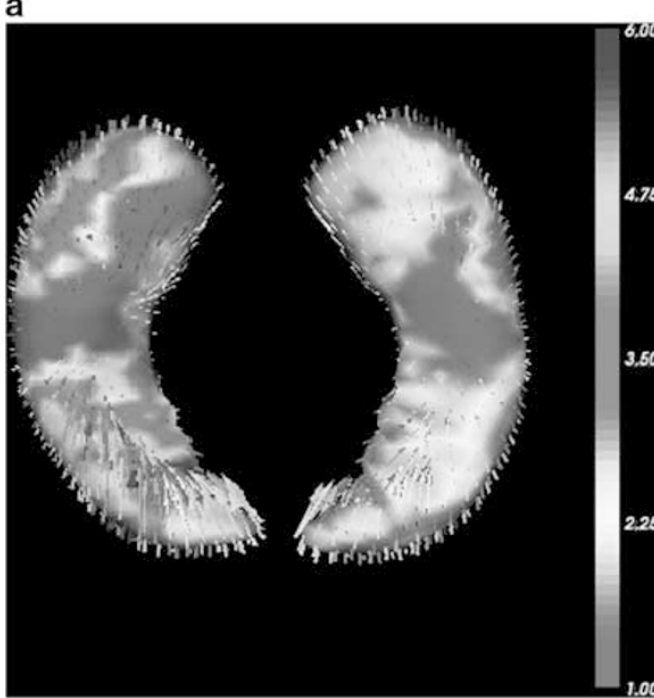

b

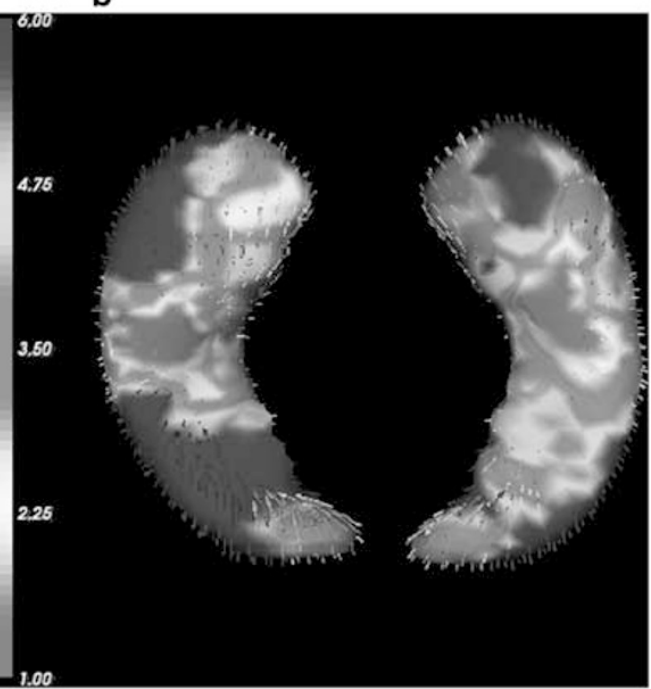

Figure 2 Results of FSL-FIRST vertex analysis of correlation of Childhood Trauma Questionnaire (CTQ)-scores with left hippocampal shape (dorsal and ventral view). The color bar indicates the values of the F-statistics: an increase from red to blue means transition from lower to higher statistical significance, whereas the length of the vectors displays means strength of correlation. For display reasons, unthresholded values are presented. The vectors indicate the direction of change: vectors pointing inwards represent local atrophy. (a) High CTQ-scores correlate significantly with local atrophy in fronto-temporal and occipito-temporal areas of the left hippocampus in major depressive disorder patients. (b) High CTQ-scores correlate significantly with local atrophy in fronto-temporal, occipito-medial, and occipito-temporal areas of the left hippocampus in the whole-sample analysis.

atrophy in MDD patients could be a function of previous maltreatment experiences rather than the diagnosis itself.

This finding bares two main consequences for the concept of morphometric anomalies in MDD. First, childhood maltreatment should be considered a powerful confounder in studies on affective disorders, and patients having suffered from maltreatment experiences might even constitute a distinct clinical subtype sharing a common set of neurobiological alterations. The present data demonstrate that the level of hippocampal atrophy in depression is strongly associated with the level of past maltreatment experiences. In consideration of these results, the impact of maltreatment on the neurobiological underpinnings of MDD might have been underestimated in former studies. 


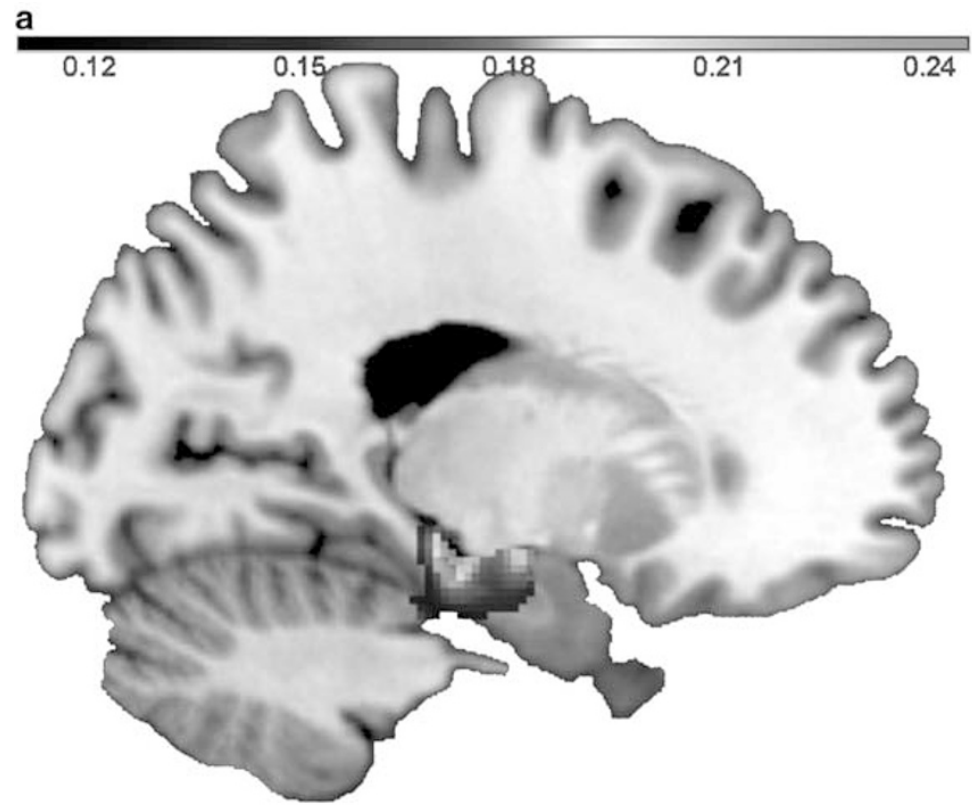

b

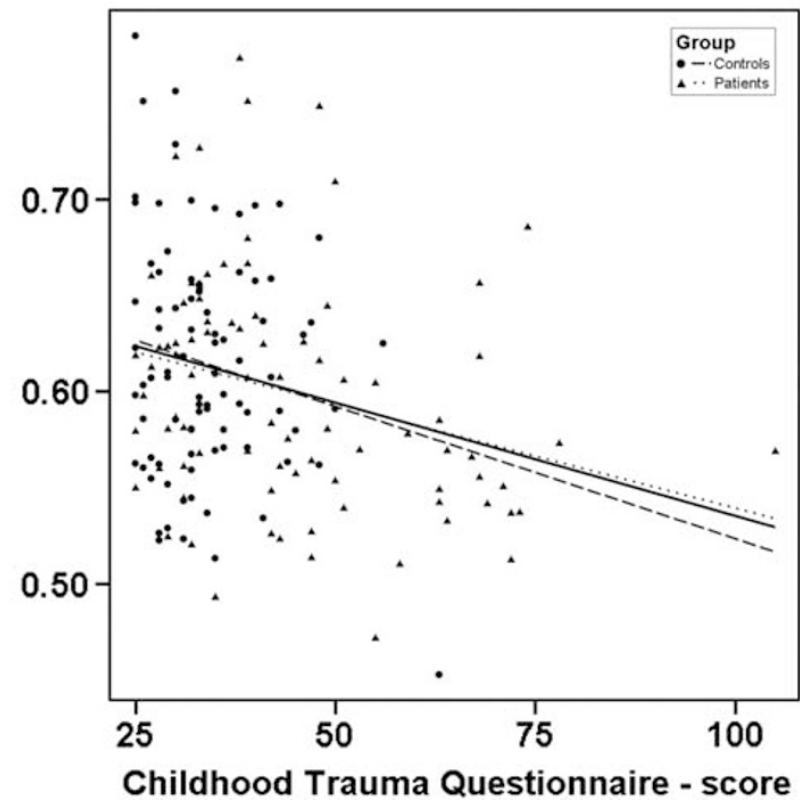

Figure 3 Effect of childhood maltreatment on hippocampal gray matter volume in the entire study sample. Left: coronal view ( $x=-\mid 4)$ depicting gray matter volume negatively associated with Childhood Trauma Questionnaire (CTQ)-scores; color bar, negative correlation coefficient $r$. Right: scatter plot depicting gray matter volume at $x=-14, y=-10, z=-24$ correlated with CTQ-scores within the entire sample. Dotted lines: regression slopes of patients and controls separately; continuous line: regression slope in the entire sample.

It has already been proposed that childhood maltreatment massively alters phenotype and associated neurobiological markers (Teicher and Samson, 2013), including brain morphology (Teicher et al, 2012), neural responsiveness (Dannlowski et al, 2012), and HPA-axis homeostasis (Heim et al, 2008). This apparent confounding of neurobiological findings in depression by early-life abuse should be avoided by accounting for this important factor in future MDD studies, not only in the context of structural neuroimaging. Similar findings are likely to be found also in functional imaging, eg, the frequently reported amygdala hyper-responsiveness in MDD (Dannlowski et al, 2007) might in the same way be a function of group differences in childhood maltreatment experience, which was repeatedly shown to be associated with amygdala hyper-responsiveness in healthy and depressed samples (Dannlowski et al, 2012; van Harmelen et al, 2013).

A second implication concerns the debate regarding stateor trait-related characteristics of hippocampal volume loss in depressed patients. Our data suggest that hippocampal volume loss is acquired by early-life experiences and might therefore constitute a trait-like risk factor for developing depression in later life. This idea of a 'limbic scar' is supported by imaging studies showing similar associations of MDD, maltreatment, and hippocampal changes (Teicher et al, 2012; Vythilingam et al, 2002). Remarkably, Rao et al (2010) recently evidenced that smaller hippocampal volumes indeed partially mediate the effect of early-life adversity on the development of MDD during longitudinal follow-up. According to this notion, hippocampal aberrations could be hypothesized to act as a connecting element in the development of severe psychopathology in maltreated individuals. Moreover, even if we did not find significant interaction effects between diagnosis, maltreatment, and hippocampal volume in the present study, interaction effects between limbic alterations, early-life adversity, and MDD might be a further explanation of these frequently observed associations as has been proposed in a recent imaging study (Frodl et al, 2010b). In addition, Gilbertson et al (2002) showed in monozygotic twins discordant for trauma exposure that, smaller hippocampi indeed constitute a risk factor for the development of stress-related psychopathology. Results of recent epigenetic studies supplement this hypothesis by revealing significantly altered methylation in genes involved in decisive pathophysiological mechanisms, such as neuronal plasticity in individuals suffering from early-life abuse (Labonté et al, 2012). Taken together, the present results indicate that hippocampal atrophy is more likely to be a pre-existing condition that renders the brain more vulnerable to the development of affective disorders, and therefore constitutes a vulnerability marker characterizing high-risk populations rather than constituting a feature of depression itself.

Further evidence for pre-existing trait-like characteristics comes from studies showing morphometric anomalies in healthy individuals at familial risk for depression (Chen et al, 2010). Hippocampal size was shown to be highly heritable (Stein et al, 2012), and several reports on genetic variations influencing shape alterations in the hippocampus support the hypothesis of hippocampal volume reductions as a predisposing trait of MDD (Frodl et al, 2008, 2010a; Stein et al, 2012). This concept of trait-related limbic aberrations further leans on studies revealing reduced hippocampal volumes in depressed to be independent of severity of depressive symptoms, age at onset, and duration of illness (Frodl et al, 2002), as it is also the case in our 
present report. In contrast, other neuroimaging studies showed hippocampal volume loss to be linked to clinical characteristics pointing to a more chronic course, eg, duration of illness and number of depressive episodes (McKinnon et al, 2009), indicating progression of hippocampal volume loss in the course of disease. Sheline et al (2003) demonstrated an association of reduced hippocampal volume and duration of untreated depressive periods. However, these findings are compatible with the concept of smaller hippocampal volumes constituting a greater risk for more severe courses of disease.

Another considerable aspect is the growing evidence for an increase of hippocampal volumes in patients undergoing different forms of psychiatric treatment, such as several weeks of antidepressant drug treatment or electroconvulsive therapy (Arnone et al, 2013; Nordanskog et al, 2010). At first sight, these state-dependent limbic alterations seem to challenge the notion of a trait-like pre-existing condition. Still, these different findings are not necessarily contradictive, as it could well be that pre-clinically existent alterations in limbic pathways are still accessible to antidepressant medication or ECT. In fact, neuronal deficits caused by stressful life events have been reported to be at least partly reversed by antidepressant medication (Duman and Aghajanian, 2012).

In summary, the present results suggest that hippocampal volume loss might preferably be characterized as a function of maltreatment rather than diagnosis, although considering that the underpinnings of these associations are likely to be of much greater complexity. Given the fact that both small hippocampal volume and history of maltreatment have been shown to predict severe courses of disease as well as lower rates of treatment response, future longitudinal studies should aim to explore the possible role of limbic alterations as a mediator in affective disorders. Thus, hippocampal morphometry might enable us to detect high-risk subjects and to provide better suitable therapy in future.

Strengths of our study include the large sample of patients and controls. Also, the results of our study were demonstrated by the use of two independent, complementary methods of subcortical morphometry (FIRST, VBM8). FSLFIRST was shown to provide precise segmentations with high reliability for the hippocampus and has successfully been utilized in MDD studies (Lai and Wu, 2011; Nugent et al, 2012), and using FSL-FIRST allowed displaying not only overall volume information, but also localized differences in shape. Furthermore, we aimed to obtain gray matter information of the hippocampus by the use of VBM, as it represents one of most commonly used instruments in morphometric studies and is highly sensitive for detecting morphometric changes in depression (Bergouignan et al, 2009) and maltreatment alike (Chaney et al, 2014; Dannlowski et al, 2012). Both methods yielded widely identical results.

A limitation of our study might be the retrospective inquiry of history and level of maltreatment by means of a self-reported measure, which might have been subject to negative recall-bias in acutely depressed patients. The inclusion of structured interviews in the assessment of past maltreatment experiences, as used in former studies, could have diminished this problem (Lenze et al, 2008; Rao et al, 2010). Although, CTQ-scores were shown to provide reliable information on past experiences of maltreatment in a large number of studies (Dannlowski et al, 2012; Teicher et al, 2012). Moreover, retrospective and prospective inquiries of childhood maltreatment were shown to provide similar results regarding the increased risk of psychopathology (Scott et al, 2012). Most of our patients were medicated with antidepressants that could have limited the validity of our results, as hippocampal size was found to be positively influenced by antidepressant medication in a recent study (Arnone et al, 2013). However, in our sample, antidepressant potency was not significantly associated with hippocampal volumes in the patient sample, indicating that medication might not have had a major role in the present study. Finally, regarding the notion that small hippocampal volumes represent a risk constellation rather than a state marker, only longitudinal designs would be able to reveal causal relationships between maltreatment, hippocampal structure, and MDD.

\section{FUNDING AND DISCLOSURE}

Professor Volker Arolt, $\mathrm{MD}, \mathrm{PhD}$, is a member of advisory boards and/or gave presentations for the following companies: Astra Zeneca, Eli Lilly, Janssen-Organon, Lundbeck, Otsuka, Servier, and Trommsdorff. Professor Peter Zwanzger, MD, has received speaker fees from Pfizer, Servier, Lilly, Astra Zeneca, and Bristol Myers Squibb, he is on the advisory board of Pfizer, is a consultant for Ironwood Pharmaceuticals, and has received funding from Astra Zeneca. These affiliations have no relevance to the work covered in the manuscript. C Konrad received fees for an educational program from esparma $\mathrm{GmbH} /$ Aristo Pharma $\mathrm{GmbH}$, Lilly Deutschland $\mathrm{GmbH}$, Servier Deutschland $\mathrm{GmbH}$, and MagVenture $\mathrm{GmbH}$, as well as travel support from Lundbeck. The study was supported by grants of the German Research Foundation (DFG) (grant FOR 2107 Project WPI to UD), Innovative Medizinische Forschung (IMF) of the Medical Faculty of Münster (DA120309 to UD, DA111107 to UD, and DA211012 to UD), and Rolf-DierichsStiftung (ZUW80037 to UD). The remaining authors declare no conflict of interest.

\section{REFERENCES}

Amico F, Meisenzahl EM, Koutsouleris NN, Reiser M, Möller H-JH-J, Frodl T (2011). Structural MRI correlates for vulnerability and resilience to major depressive disorder. J Psychiatry Neurosci 36: 15-22.

Arnone D, McIntosh AM, Ebmeier KP, Munafò MR, Anderson IM (2012a). Magnetic resonance imaging studies in unipolar depression: systematic review and meta-regression analyses. Eur Neuropsychopharmacol 22: 1-16.

Arnone D, McKie S, Elliott R, Juhasz G, Thomas EJ, Downey D et al (2013). State-dependent changes in hippocampal grey matter in depression. Mol Psychiatry 18: 1265-1272.

Beck AT, Steer RA. Beck Depression Inventory: Manual. Psychological Corporation: San Antonio, TX, USA, Harcourt Brace Jovanovich: New York, NY, USA (1987).

Bergouignan L, Chupin M, Czechowska Y, Kinkingnéhun S, Lemogne C, Bastard G Le et al (2009). Can voxel based morphometry, manual segmentation and automated segmentation equally detect hippocampal volume differences in acute depression? Neuroimage 45: 29-37. 
Bernstein DP, Fink L, Handelsman L, Foote J, Lovejoy M, Wenzel K et al (1994). Initial reliability and validity of a new retrospective measure of child abuse and neglect. Am J Psychiatry 151: $1132-1136$

Chaney A, Carballedo A, Amico F, Fagan A, Skokauskas N, Meaney $\mathrm{J}$ et al (2014). Effect of childhood maltreatment on brain structure in adult patients with major depressive disorder and healthy participants. J Psychiatry Neurosci 39: 50-59.

Chen MC, Hamilton JP, Gotlib IH (2010). Decreased hippocampal volume in healthy girls at risk of depression. Arch Gen Psychiatry 67: 270-276.

Cole J, Costafreda SG, McGuffin P, Fu CHY (2011). Hippocampal atrophy in first episode depression: a meta-analysis of magnetic resonance imaging studies. J Affect Disord 134: 483-487.

Covic M, Karaca E, Lie DC (2010). Epigenetic regulation of neurogenesis in the adult hippocampus. Heredity 105: 122-134.

Cusi AM, Nazarov A, Holshausen K, Macqueen GM, McKinnon MC (2012). Systematic review of the neural basis of social cognition in patients with mood disorders. J Psychiatry Neurosci 37: 154-169.

Dannlowski U, Ohrmann P, Bauer J, Kugel H, Arolt V, Heindel W et al (2007). Amygdala reactivity to masked negative faces is associated with automatic judgmental bias in major depression: a 3T fMRI study. J Psychiatry Neurosci 32: 423-429.

Dannlowski U, Ohrmann P, Konrad C, Domschke K, Bauer J, Kugel $\mathrm{H}$ et al (2009). Reduced amygdala-prefrontal coupling in major depression: association with MAOA genotype and illness severity. Int J Neuropsychopharmacol 12: 11-22.

Dannlowski U, Stuhrmann A, Beutelmann V, Zwanzger P, Lenzen T, Grotegerd D et al (2012). Limbic scars: long-term consequences of childhood maltreatment revealed by functional and structural magnetic resonance imaging. Biol Psychiatry 71: 286-293.

Duman RS, Aghajanian GK (2012). Synaptic dysfunction in depression: potential therapeutic targets. Science 338: 68-72.

Edmiston EE, Wang F, Mazure CM, Guiney J, Sinha R, Mayes LC et al (2011). Corticostriatal-limbic gray matter morphology in adolescents with self-reported exposure to childhood maltreatment. Arch Pediatr Adolesc Med 165: 1069-1077.

Eriksson PS, Perfilieva E, Björk-Eriksson T, Alborn a M, Nordborg C, Peterson DA et al (1998). Neurogenesis in the adult human hippocampus. Nat Med 4: 1313-1317.

Frodl T, Koutsouleris NN, Bottlender R, Born C, Jäger $M$, Mörgenthaler $\mathrm{M}$ et al (2008). Reduced gray matter brain volumes are associated with variants of the serotonin transporter gene in major depression. Mol Psychiatry 13: 1093-1101.

Frodl T, Meisenzahl EM, Zetzsche T, Born C, Groll C, Jäger M et al (2002). Hippocampal changes in patients with a first episode of major depression. Am J Psychiatry 159: 1112-1118.

Frodl T, Reinhold E, Koutsouleris NN, Donohoe G, Bondy B, Reiser $\mathrm{M}$ et al (2010a). Childhood stress, serotonin transporter gene and brain structures in major depression. Neuropsychopharmacology 35: 1383-1390.

Frodl T, Reinhold E, Koutsouleris NN, Reiser M, Meisenzahl EM (2010b). Interaction of childhood stress with hippocampus and prefrontal cortex volume reduction in major depression. J Psychiatr Res 44: 799-807.

Gilbert R, Widom CS, Browne K, Fergusson D, Webb E, Janson S (2009). Burden and consequences of child maltreatment in high-income countries. Lancet 373: 68-81.

Gilbertson MW, Shenton ME, Ciszewski A, Kasai K, Lasko NB, Orr SP et al (2002). Smaller hippocampal volume predicts pathologic vulnerability to psychological trauma. Nat Neurosci 5: 1242-1247.

Hamilton M (1960). A rating scale for depression. J Neurol Neurosurg Psychiatry 23: 56-62.

Heim CM, Newport DJ, Mletzko TC, Miller AH, Nemeroff CB (2008). The link between childhood trauma and depression: insights from HPA axis studies in humans. Psychoneuroendocrinology 33: 693-710.

Labonté B, Suderman M, Maussion G, Navaro L, Yerko V, Mahar I et al (2012). Genome-wide epigenetic regulation by early-life trauma. Arch Gen Psychiatry 69: 722-731.

Lai C-H, Wu Y-T (2011). Duloxetine's modest short-term influences in subcortical structures of first episode drug-naive patients with major depressive disorder and panic disorder. Psychiatry Res 194: 157-162.

Lenze SN, Xiong C, Sheline YI (2008). Childhood adversity predicts earlier onset of major depression but not reduced hippocampal volume. Psychiatry Res 162: 39-49.

MacQueen GM, Frodl T (2011). The hippocampus in major depression: evidence for the convergence of the bench and bedside in psychiatric research? Mol Psychiatry 16: 252-264.

McKinnon MCM, Yucel K, Nazarov A, MacQueen GM (2009). A meta-analysis examining clinical predictors of hippocampal volume in patients with major depressive disorder. J Psychiatry Neurosci 34: 41-54.

Nanni V, Uher R, Danese A (2012). Childhood maltreatment predicts unfavorable course of illness and treatment outcome in depression: a meta-analysis. Am J Psychiatry Psychiatry 169: 141-151.

Nordanskog P, Dahlstrand U, Larsson MR, Larsson E-M, Knutsson L, Johanson A (2010). Increase in hippocampal volume after electroconvulsive therapy in patients with depression: a volumetric magnetic resonance imaging study. J ECT 26: 62-67.

Nugent AC, Luckenbaugh DA, Wood SE, Bogers W, Zarate CA Jr, Drevets WC (2012). Automated subcortical segmentation using FIRST: test-retest reliability, interscanner reliability, and comparison to manual segmentation. Hum Brain Mapp 34: 2313-2329.

Rao U, Chen L-A, Bidesi AS, Shad MU, Thomas MA, Hammen CL (2010). Hippocampal changes associated with early-life adversity and vulnerability to depression. Biol Psychiatry 67: 357-364.

Sackeim HA (2001). The definition and meaning of treatmentresistant depression. J Clin Psychiatry 62(Suppl 1): 10-17.

Scott KM, McLaughlin KA, Smith DA, Ellis PM (2012). Childhood maltreatment and DSM-IV adult mental disorders: comparison of prospective and retrospective findings. Br J Psychiatry 200: 469-475.

Sheline YI, Gado MH, Kraemer HC (2003). Untreated depression and hippocampal volume loss. Am J Psychiatry 160: 1516-1518.

Stein JL, Medland SE, Vasquez AA, Hibar DP, Senstad RE, Winkler AM et al (2012). Identification of common variants associated with human hippocampal and intracranial volumes. Nat Genet 44: 552-561.

Stuhrmann A, Suslow T, Dannlowski U (2011). Facial emotion processing in major depression: a systematic review of neuroimaging findings. Biol Mood Anxiety Disord 1: 10.

Surguladze S, Brammer MJ, Keedwell P, Giampietro V, Young AW, Travis MJ et al (2005). A differential pattern of neural response toward sad versus happy facial expressions in major depressive disorder. Biol Psychiatry 57: 201-209.

Suslow T, Konrad C, Kugel H, Rumstadt D, Zwitserlood P, Schöning $S$ et al (2010). Automatic mood-congruent amygdala responses to masked facial expressions in major depression. Biol Psychiatry 67: 155-160.

Teicher MH, Anderson CM, Polcari A (2012). Childhood maltreatment is associated with reduced volume in the hippocampal subfields CA3, dentate gyrus, and subiculum. Proc Natl Acad Sci USA 109: E563-E572.

Teicher MH, Dumont NL, Ito Y, Vaituzis C, Giedd JN, Andersen SL (2004). Childhood neglect is associated with reduced corpus callosum area. Biol Psychiatry 56: 80-85.

Teicher MH, Samson JA (2013). Childhood maltreatment and psychopathology: a case for ecophenotypic variants as clinically and neurobiologically distinct subtypes. Am J Psychiatry 170: 1114-1133. 
van Harmelen A-L, van Tol M-J, Demenescu LR, van der Wee NJA, Veltman DJ, Aleman A et al (2013). Enhanced amygdala reactivity to emotional faces in adults reporting childhood emotional maltreatment. Soc Cogn Affect Neurosci 8: 362-369.

van Harmelen A-L, van Tol M-J, van der Wee NJ, Veltman DJ, Aleman A, Spinhoven P et al (2010). Reduced medial prefrontal cortex volume in adults reporting childhood emotional maltreatment. Biol Psychiatry 68: 832-838.

Vythilingam M, Heim CM, Newport J, Miller AH, Anderson E, Bronen $\mathrm{R}$ et al (2002). Childhood trauma associated with smaller hippocampal volume in women with major depression. Am J Psychiatry 159: 2072-2080.

Wang Y-C, Huang C-C, Hsu K-S (2010). The role of growth retardation in lasting effects of neonatal dexamethasone treatment on hippocampal synaptic function. PLoS One 5: e12806.

Wittchen H-U, Wunderlich U, Gruschwitz S, Zaudig M. SKID-1. Strukturiertes Klinisches Interview für DSM-IV. Hogrefe: Göttingen (1997).

World Health Organization. Mental health: New Understanding, New Hope. World Health Organization: Geneva, Switzerland (2001).

Supplementary Information accompanies the paper on the Neuropsychopharmacology website (http://www.nature.com/npp) 